\title{
QUEEN'S
UNIVERSITY
BELFAST
}

\section{Nonequilibrium generalised Langevin equation for the calculation of heat transport properties in model 1D atomic chains coupled to two 3D thermal baths}

Ness, H., Stella, L., Lorenz, C. D., \& Kantorovich, L. (2017). Nonequilibrium generalised Langevin equation for the calculation of heat transport properties in model 1D atomic chains coupled to two 3D thermal baths. Journal of Chemical Physics, 146(16), [164103]. https://doi.org/10.1063/1.4981816

Published in:

Journal of Chemical Physics

Document Version:

Publisher's PDF, also known as Version of record

Queen's University Belfast - Research Portal:

Link to publication record in Queen's University Belfast Research Portal

\section{Publisher rights}

Copyright 2017 the authors.

This work is made available online in accordance with the publisher's policies. Please refer to any applicable terms of use of the publisher.

\section{General rights}

Copyright for the publications made accessible via the Queen's University Belfast Research Portal is retained by the author(s) and / or other copyright owners and it is a condition of accessing these publications that users recognise and abide by the legal requirements associated with these rights.

Take down policy

The Research Portal is Queen's institutional repository that provides access to Queen's research output. Every effort has been made to ensure that content in the Research Portal does not infringe any person's rights, or applicable UK laws. If you discover content in the

Research Portal that you believe breaches copyright or violates any law, please contact openaccess@qub.ac.uk. 


\section{Nonequilibrium generalised Langevin equation for the calculation of heat transport properties in model 1D atomic chains coupled to two 3D thermal baths}

H. Ness, L. Stella, C. D. Lorenz, and L. Kantorovich

Citation: The Journal of Chemical Physics 146, 164103 (2017); doi: 10.1063/1.4981816

View online: http://dx.doi.org/10.1063/1.4981816

View Table of Contents: http://aip.scitation.org/toc/jcp/146/16

Published by the American Institute of Physics

\section{Articles you may be interested in}

Perspective: Dissipative particle dynamics

The Journal of Chemical Physics 146, 150901150901 (2017); 10.1063/1.4979514

Atomistic simulations of wetting properties and water films on hydrophilic surfaces

The Journal of Chemical Physics 146, 164705164705 (2017); 10.1063/1.4979847

Stochastic simulation of reaction-diffusion systems: A fluctuating-hydrodynamics approach

The Journal of Chemical Physics 146, 124110124110 (2017); 10.1063/1.4978775

Molecular dynamics simulation of nanobubble nucleation on rough surfaces

The Journal of Chemical Physics 146, 164704164704 (2017); 10.1063/1.4981788

A Gaussian theory for fluctuations in simple liquids

The Journal of Chemical Physics 146, 134507134507 (2017); 10.1063/1.4979659

Energy-based truncation of multi-determinant wavefunctions in quantum Monte Carlo

The Journal of Chemical Physics 146, 164101164101 (2017); 10.1063/1.4981527

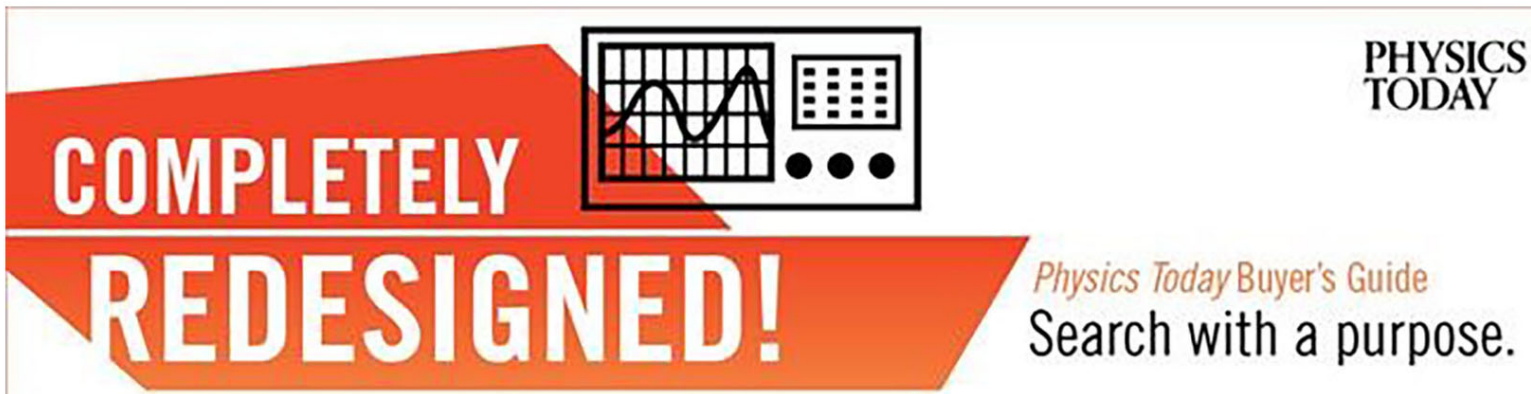




\title{
Nonequilibrium generalised Langevin equation for the calculation of heat transport properties in model 1D atomic chains coupled to two 3D thermal baths
}

\author{
H. Ness, ${ }^{1, a)}$ L. Stella, ${ }^{2}$ C. D. Lorenz, ${ }^{1}$ and L. Kantorovich ${ }^{1}$ \\ ${ }^{1}$ Department of Physics, Faculty of Natural and Mathematical Sciences, King's College London, Strand, \\ London WC2R 2LS, United Kingdom \\ ${ }^{2}$ Atomistic Simulation Centre, School of Mathematics and Physics, Queen's University Belfast, University Road, \\ Belfast BT7 1NN, Northern Ireland, United Kingdom
}

(Received 25 January 2017; accepted 7 April 2017; published online 24 April 2017)

\begin{abstract}
We use a generalised Langevin equation scheme to study the thermal transport of low dimensional systems. In this approach, the central classical region is connected to two realistic thermal baths kept at two different temperatures [H. Ness et al., Phys. Rev. B 93, 174303 (2016)]. We consider model Al systems, i.e., one-dimensional atomic chains connected to three-dimensional baths. The thermal transport properties are studied as a function of the chain length $N$ and the temperature difference $\Delta T$ between the baths. We calculate the transport properties both in the linear response regime and in the non-linear regime. Two different laws are obtained for the linear conductance versus the length of the chains. For large temperatures $(T \gtrsim 500 \mathrm{~K})$ and temperature differences $(\Delta T \gtrsim 500 \mathrm{~K})$, the chains, with $N>18$ atoms, present a diffusive transport regime with the presence of a temperature gradient across the system. For lower temperatures $(T \lesssim 500 \mathrm{~K})$ and temperature differences $(\Delta T \lesssim 400 \mathrm{~K})$, a regime similar to the ballistic regime is observed. Such a ballistic-like regime is also obtained for shorter chains $(N \leq 15)$. Our detailed analysis suggests that the behaviour at higher temperatures and temperature differences is mainly due to anharmonic effects within the long chains. Published by AIP Publishing. [http://dx.doi.org/10.1063/1.4981816]
\end{abstract}

\section{INTRODUCTION}

Understanding the physical properties of low-dimensional thermal transport is an active field of research since such properties are often counter intuitive and present intriguing features. ${ }^{1-6}$ A recent review on the subject can be found in Ref. 7. For instance, some one-dimensional (1D) models violate the well-known Fourier law of heat transport, while some others do not.

It has been known, since the seminal work of Rieder et al. ${ }^{8}$ that in 1D homogeneous harmonic systems (also referred to as integrable systems), the thermal conductivity diverges in the thermodynamic limit. No temperature gradient is formed in the bulk of the system, since the dominating energy "carriers" are not scattered and propagate ballistically. A large variety of harmonic (integrable) classical ${ }^{8-17}$ and quantum ${ }^{10,13,18-23}$ systems have been studied using analytical and/or numerical approaches. All these studies show that there is no temperature gradient inside the system (except for small regions in the vicinity of the contacts between the central system and the baths). One usually obtains a constanttemperature profile ${ }^{8-12,15,17-23}$ in the central system around the average temperature $T_{\mathrm{av}}=\left(T_{L}+T_{R}\right) / 2$.

On the other hand, in classical or quantum non-integrable systems, a temperature gradient is formed inside the system. The temperature gradient is uniform, and the heat

\footnotetext{
a)Electronic mail: herve.ness@kcl.ac.uk
}

conductivity is finite. The transport is said to be diffusive and these systems obey Fourier's law. In order to obtain a diffusive transport regime, one has to introduce any form of anharmonic effects in the system. $1,10,12-14,16,17,22,25,26,28-37$ Diffusive transport is also obtained when extra local stochastic processes $^{9,10,15,17,18,25,31,40}$ or extra collision processes ${ }^{41,42}$ are introduced. A vibrational mode coupling in classical systems ${ }^{43}$ is also responsible for diffusive transport.

The introduction of configurational defects ${ }^{1,24,38}$ or disorder $9,15,24,30,39$ in harmonic systems can also lead to diffusive transport, with the buildup of a temperature gradient across the system. Defects and disorder introduce some form of localisation of the vibration modes ${ }^{26,27}$ which do not favour ballistic transport. ${ }^{26}$

In the previously mentioned studies, the system is either ballistic or diffusive. However it is very important to understand if a crossover between the two regimes can be obtained as it has been observed experimentally in a wide range of low-dimensional systems (from carbon nanotubes, ${ }^{44}$ graphene nanoribbons, ${ }^{45}$ nanowires,${ }^{46}$ and polymer nanofibres ${ }^{47}$ ). From the theoretical point of view, the ballistic to diffusive crossover has been studied phenomenologically by introducing and tuning a viscosity-like coefficient (in a simple Langevin-like dynamics) on each atom in the system, ${ }^{17,48}$ by modifying the interatomic potential, ${ }^{13}$ or by introducing a simple description of phonon-phonon interaction. ${ }^{49} \mathrm{~A}$ single scheme that can describe both regimes is central and crucially needed to understand the origin of the crossover. Recently, a unified microscopy formalism to study the ballistic-to-diffusive 
crossover was provided in Ref. 50 on the basis of a scaling ansatz for model systems.

In this manuscript, we present an application of our recently developed Generalised Langevin Equation (GLE) method to the study of the heat transport properties of lowdimensional systems. Within this single GLE scheme, we simulate the dissipative dynamics of model $\mathrm{Al}$ systems. Our main objective is to determine if different transport regimes can be established depending on the chain's length and temperatures involved.

We consider 1D atomic chains connected to two realistic thermal 3D baths kept at temperatures $T_{L}$ and $T_{R}$. By realistic it is meant that (1) the bath is described at the atomic level, with a proper 3D structure, (2) the coupling between the system and the baths obtained in our GLE approach is not simply given by some arbitrary constant(s), and (3) the inter-atomic interaction is given by a $\mathrm{N}$-body type interaction designed in materials science and not by a model pairwise potential. Such a N-body potential gives a realistic description of the covalent bonding between atoms in metallic systems which is not well described by pair-wise potentials. Therefore, we use the Embedded Atom Method (EAM) ${ }^{51-53}$ to describe the interatomic potential between the $\mathrm{Al}$ atoms in the $1 \mathrm{D}$ chains and between the chains and the two thermal baths. The only "free parameters" that can be changed are the length $N$ of the 1D chains and the temperatures $T_{L}$ and $T_{R}$ of the baths. We calculate the transport properties of the 1D atomic chains in both the linear response regime and at full nonequilibrium conditions. We study the temperature and length dependence of the linear heat conductance and of the nonequilibrium heat current. We find different transport regimes, which appear to be more characteristic of either the diffusive or ballistic regime, depending on the temperatures and temperature differences and on the length of the chains. We analyse the results in terms of anharmonic versus harmonic effects in the effective interatomic potential.

\section{METHOD AND SYSTEMS}

To study the thermal transport of 1D atomic chains, we used our recently developed GLE scheme. ${ }^{54,55}$ The method and its implementation in the molecular dynamics package LAMMPS $^{56}$ have been well documented in recent papers. ${ }^{57,58}$ The extension of the original GLE scheme to situations where the central system is interacting with several independent baths has been recently given in Ref. 59. We call it the GLE2B scheme. We stress that this method, at least in principle, enables one to obtain an exact solution of the problem of heat transport in the classical case. The method is based on a physically realistic picture of infinite leads kept at equilibrium (at corresponding temperatures) and an arbitrary central region which interacts and exchanges heat with the leads. The only approximations made are that the leads are treated as harmonic baths and the interaction between the leads and the central system is linear in terms of displacements of the atoms in the leads; the central system is treated without any approximations and can be arbitrarily anharmonic.

In a recent paper, ${ }^{59}$ we have discussed the advantages of the GLE-2B approach over other more conventional thermostatting approaches such as Nose-Hoover or simple Langevin thermostats. Here, we use our GLE-2B approach as it does not rely on the use of adjustable parameter(s) to describe the relaxation processes in the baths. In Ref. 59, we have shown that depending upon the value used for such parameters, one can obtain completely different physical results. In the present work, we want to study exclusively the influence of the length of the system and the baths' temperatures on the thermal transport properties.

We now briefly recall the main "ingredients" of the method. We consider a central system (the 1D atomic chain) with a general Hamiltonian dynamics for the positions $r_{i \alpha}$ and momenta $p_{i \alpha}$ degrees of freedom (DOF) associated with atom $i$ (mass $m_{i}$ ) and Cartesian coordinates $\alpha=x, y, z$. The central system is connected to two $(v=1,2)$ harmonic baths, with DOF $u_{b_{v}}$ and $\dot{u}_{b_{v}}$, via coupling $\sum_{b_{v}} \mu_{l_{v}} f_{b_{v}}(\mathbf{r}) u_{b_{v}}$. The coupling is linear with respect to the atomic displacement $u_{b_{v}}$ $\left(b_{v} \equiv l_{v} \gamma\right)$ of the atom $l_{v}(\gamma=x, y, z)$, in bath $v$, around its equilibrium position. $\mu_{l_{v}}$ is the mass of atom $l_{v}$. The coupling force $\mu_{l_{v}} f_{b_{v}}(\mathbf{r})$ between the central system and the bath $v$ is arbitrary with respect to the central system DOF $r_{i \alpha}$.

By solving Newton's equations of motion for all the DOFs, and integrating out the baths' DOFs, one obtains the following "embedded" dissipative dynamics for the DOF of the central system: ${ }^{54,59}$

$m_{i} \ddot{r}_{i \alpha}=\mathcal{F}_{i \alpha}-\int_{-\infty}^{t} \mathrm{~d} t^{\prime} \sum_{\nu, i^{\prime} \alpha^{\prime}} K_{i \alpha, i^{\prime} \alpha^{\prime}}^{(v)}\left(t, t^{\prime} ; \mathbf{r}\right) \dot{r}_{i^{\prime} \alpha^{\prime}}\left(t^{\prime}\right)+\sum_{\nu} \eta_{i \alpha}^{(v)}(t ; \mathbf{r})$.

The total force $\mathcal{F}_{i \alpha}$ acting on atom $i$ of the central system (in direction $\alpha$ ) arises not only from the interaction between atoms in the central system, but also from the interaction between these atoms and the atoms of the harmonic baths which are kept fixed at their equilibrium positions. The force $\mathcal{F}_{i \alpha}$ also contains a polaronic-like term which reflects the fact that, due to the coupling between the central system and the baths, the harmonic oscillators of the baths are displaced. ${ }^{54,57-59}$

Eq. (1) also contains a generalised memory kernel which depends on both times $t$ and $t^{\prime}$ separately (i.e., not on their difference) and also on the spatial coordinates (DOFs) $\mathbf{r}$ $=\left(r_{i \alpha}\right)$ of all atoms of the central system. The memory kernel is given by

$$
\begin{aligned}
K_{i \alpha, i^{\prime} \alpha^{\prime}}^{(v)}\left(t, t^{\prime} ; \mathbf{r}\right)= & \sum_{b_{v}, b_{\nu}^{\prime}} \sqrt{\mu_{l_{\nu}} \mu_{l_{\nu}^{\prime}}} g_{i \alpha, b_{\nu}}(\mathbf{r}(t)) \\
& \times \prod_{b_{v}, b_{v}^{\prime}}\left(t-t^{\prime}\right) g_{i^{\prime} \alpha^{\prime}, b_{\nu}^{\prime}}\left(\mathbf{r}\left(t^{\prime}\right)\right),
\end{aligned}
$$

where $g_{i \alpha, b_{\nu}}$ is the derivative of the coupling force $g_{i \alpha, b_{\nu}}$ $=\partial f_{b_{v}}(\mathbf{r}) / \partial r_{i \alpha}$ and the polarization matrix $\Pi_{b_{v}, b_{v}^{\prime}}\left(t-t^{\prime}\right)$ is related to the harmonic dynamical matrix of the bath $v .^{54,57-59}$ In the energy representation, the polarization matrix $\Pi_{b_{v}, b_{v}^{\prime}}(\omega)$ represents the vibrational density of states of the corresponding bath. ${ }^{54,57-59}$ Finally, the terms $\eta_{i \alpha}^{(v)}$ contain all the information about the initial conditions of the bath $v$ DOFs.

By considering these initial conditions as random processes, the corresponding stochastic forces $\eta_{i \alpha}^{(v)}$ can be described by a multi-dimensional Gaussian stochastic process 
with correlation functions, ${ }^{54,57}$

$$
\begin{aligned}
\left\langle\eta_{i \alpha}^{(v)}(t ; \mathbf{r})\right\rangle & =0, \\
\left\langle\eta_{i \alpha}^{(v)}(t ; \mathbf{r}) \eta_{i^{\prime} \alpha^{\prime}}^{\left(\nu^{\prime}\right)}\left(t^{\prime} ; \mathbf{r}\right)\right\rangle & =\delta_{\nu v^{\prime}} k_{B} T_{\nu} K_{i \alpha, i^{\prime} \alpha^{\prime}}^{(v)}\left(t, t^{\prime} ; \mathbf{r}\right),
\end{aligned}
$$

and consequently Eq. (1) corresponds now to a generalised Langevin equation for the central system DOFs, with a nonMarkovian memory kernel and coloured noise.

Eq. (1) can be efficiently solved numerically by introducing a set of auxiliary DOFs (aDOF) $\left\{s_{v, 1}^{\left(k_{\nu}\right)}(t), s_{v, 2}^{\left(k_{\nu}\right)}(t)\right\}$ (the superscript $k_{v}$ is used to count the aDOFs) ${ }^{57,60-65}$ and by mapping the polarization matrix onto a specific analytical form, ${ }^{57}$

$$
\Pi_{b_{v}, b_{v}^{\prime}}\left(t-t^{\prime}\right) \rightarrow \sum_{k_{v}} A_{b_{v} b_{v}^{\prime}}^{\left(k_{k}\right)} e^{-\left|t-t^{\prime}\right| / \tau_{k_{v}}} \cos \left(\omega_{k_{v}}\left|t-t^{\prime}\right|\right) .
$$

Each pair of aDOF $\left\{s_{v, 1}^{\left(k_{v}\right)}, s_{v, 2}^{\left(k_{v}\right)}\right\}$ is associated with the corresponding mapping coefficients $\left\{\tau_{k_{v}}, \omega_{k_{v}}, c_{b_{v}}^{\left(k_{\nu}\right)}\right\}$. Then it can be shown that solving Eq. (1) is equivalent to solving an extended set of Langevin equations, for the central system DOFs and the aDOFs, as a multivariate Markovian process, where all the DOFs are independent Wiener stochastic processes with (white noise) correlation functions. ${ }^{57-59}$

The systems we consider are 1D atomic chains (of length $N$ ) connected to 3D thermal baths as shown in Figure 1. The electronic transport properties of similar $\mathrm{Al}$ nanowires have been studied some decades ago. ${ }^{66-68}$ It is now interesting to study their thermal transport properties using our method. We have taken the embedded atom method ${ }^{51}$ to model the metallic Al system. The tabulated interatomic potential, provided by the NIST Interatomic Potential Repository Project, ${ }^{52}$ is a typical non-pairwise potential.

It is important to note that, when using realistic interatomic interaction potential, the model goes beyond the balland-spring (with nearest-neighbour interaction) toy models. In Fig. 1 we show that more than two end-atoms of the 1D chains are coupled directly to the thermal baths. In the present case,

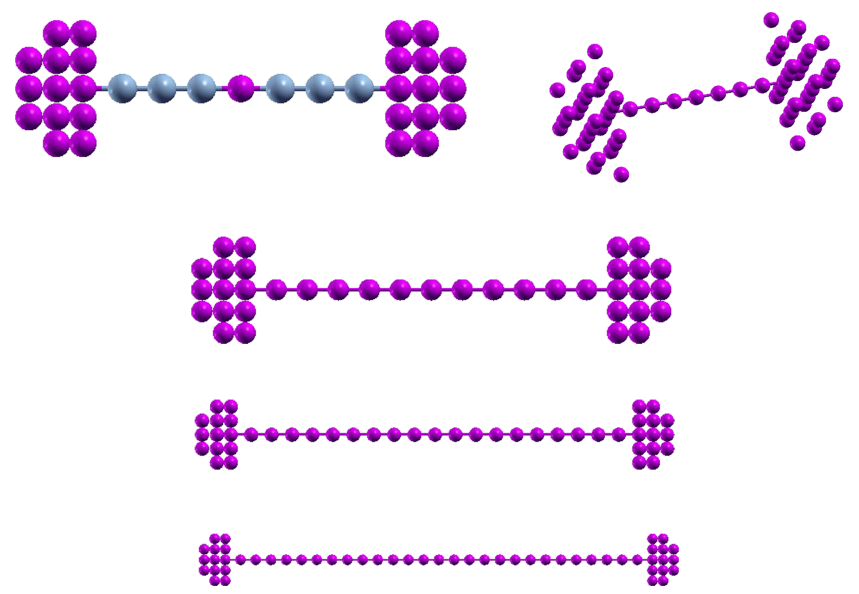

FIG. 1. Schematic representation of the different one-dimensional Al chains (length $N=7,11,19,27$ from top to bottom) connected to two threedimensional $L$ and $R$ baths (each made of $30 \mathrm{Al}$ atoms). The baths shown here are in fact what is called the reduced bath regions. ${ }^{58,59}$ These regions contain atoms $b_{v}$ which interact with atoms $i \alpha$ of the central region via nonzero matrix elements $g_{i \alpha, b_{\gamma}}$. The EAM is used for the interatomic interaction. The three first (last) atoms in the chains (shown in a different color in the top left corner) are the atoms interacting directly with the left (right) bath respectively. the first (last) 3 atoms of the 1D chains are coupled directly to the left (right) thermal bath. Furthermore the coupling of these atoms to the baths is given by the matrix elements $g_{i \alpha, b_{v}}$ which are not constants throughout the GLE dynamics. These matrix elements are explicitly dependent on the positions of the atoms in the 1D chains and change accordingly along the GLE dynamics.

Finally, the thermal baths have their own specific spectral signatures, given by the polarisation matrix $\Pi_{b_{v}, b_{v}^{\prime}}(\omega)$ (obtained from the Fourier transform of $\Pi_{b_{v}, b_{v}^{\prime}}\left(t-t^{\prime}\right)$ ). The frequency dependence of $\Pi_{b_{v}, b_{\nu}^{\prime}}(\omega)$ is not trivial and depends on the very nature (atomic configuration, chemical nature, etc.) of the corresponding bath described at the atomic level. Since in our approach we are dealing with a realistic description of the baths, it is not straightforward to determine if our baths are ohmic (sub- or super-ohmic) as is usually done in other simulations of less realistic models.

\section{RESULTS}

We focus our study on the steady state only and consider chains of length $N=7,9,11,13,15,19,21,23,25,27$ atoms. To obtain interesting low-dimensional transport properties, the dynamics of the atoms in the chains is constrained to the 1D motion along the chain axis ( $x$-direction) by imposing the condition $p_{i y}=p_{i z}=0$ for every atom $i$ in the chain.

We calculate the heat current $J_{\text {heat }}\left(N, T_{L}, T_{R}\right)$ from the time derivative of the lead Hamiltonian, which results in the sum, over the chain atoms, of the products of their velocity and the corresponding force ${ }^{3,13}$ (see Appendix A for further details).

The GLE-2B simulations are typically performed for $300 \mathrm{ps}$ ( 150000 time steps with $\Delta t=2 \mathrm{fs}$ ). The steady state is reached after around $100 \mathrm{ps}$. We calculate a time average of the heat current $J_{\text {heat }}\left(N, T_{L}, T_{R}\right)$ over the time range between 200 and 300 ps where the system has reached the steady state (Appendix A). Furthermore, for information, all the results obtained for the heat current $J_{\text {heat }}$ flowing across the chains

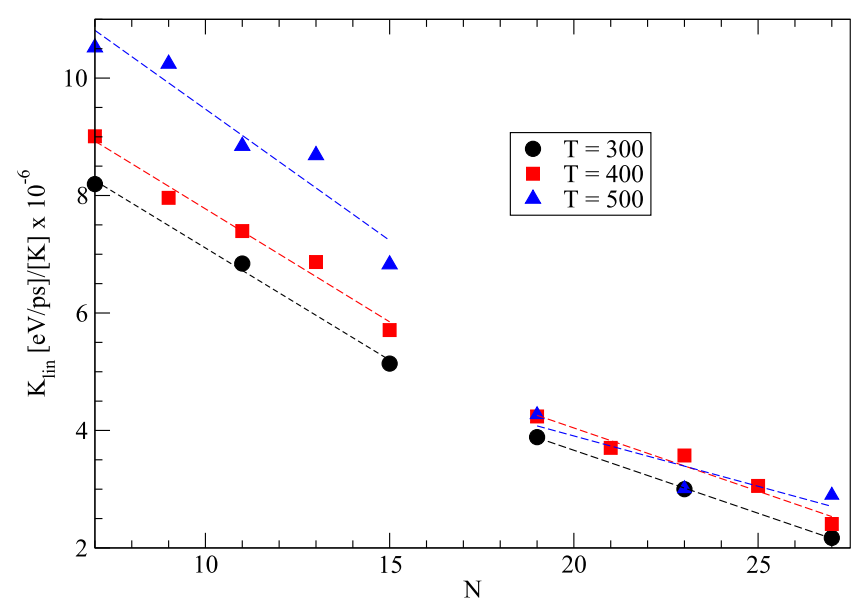

FIG. 2. Linear thermal conductance $K_{\text {lin }}$ for the $1 \mathrm{D}$ chains of different length $N$ and for different temperatures $T$. (Note that, in the linear regime, $\Delta T \rightarrow 0$ and $T_{L} \rightarrow T_{R} \equiv T$.) It appears that the length dependence of $K_{\text {lin }}$ is not the same for the shorter chains (i.e., $N<16)$ and for the longest chains $(N>18)$. The lines are guides for the eye based on the best linear fit of the calculated points. 


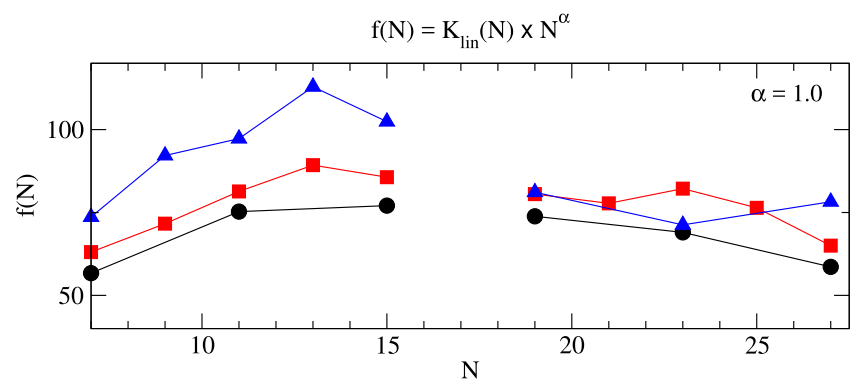

FIG. 3. The function $f(N)=K_{\operatorname{lin}}(N, T) N^{\alpha}$ is plotted for $\alpha=1.0$ for the different temperatures. It appears that the function $f(N)$ is more constant for the longer chains than for the shorter chains. Hence, for the longer chains, we have $K_{\operatorname{lin}}(N) \propto 1 / N$, while for the shorter chains $K_{\operatorname{lin}}(N)$ does not follow the same length dependence. Note that the color of the lines corresponds to the temperatures given in Fig. 2. The units of $f(N)$ are the same as the unit of $K_{\text {lin }}$ in Fig. 2, i.e., $\times 10^{-6}[\mathrm{eV} / \mathrm{ps} \mathrm{K}]$.

and for all set of temperatures $T_{L}$ and $T_{R}$ are given in Figure 9 of Appendix B.

From the slope of $J_{\text {heat }}(N, T, \Delta T)$ at a small temperature difference, ${ }^{13}$ we extract the value of the linear thermal conductance $K_{\text {lin }}(N, T)=\lim _{\Delta T \rightarrow 0} J_{\text {heat }}(N, T, \Delta T) / \Delta T$. Figure 2 shows the linear thermal conductance $K_{\text {lin }}$ versus the length $N$ for different temperatures $T_{L} \rightarrow T_{R}$. We can observe two different regimes for the behaviour of $J_{\text {heat }}$ versus $N$ : one for short chains (typically $N<16$ ) and the other for longer chains (typically $N>18$ ).

For the longer chains, the length dependence of $K_{\text {lin }}$ follows quite well the typical $1 / N$ power law as shown in Fig. 3. We have calculated the function $f(N)=K_{\operatorname{lin}}(N) N^{\alpha}$ for different values of $\alpha \sim 1$, and the results for $\alpha=1$ are shown in Fig. 3 for the different temperatures corresponding to Fig. 2. Qualitatively speaking, the function $f(N)$ is more flat for the longer chains $(N>18)$, i.e., $K_{\text {lin }}$ follows a power law in $1 / N^{\alpha}$ with $\alpha \sim 1$ (diffusive Fourier law). For the shorter chains $(N \leq 15)$, the function $f(N)$ clearly presents a stronger dependence on $N$ (i.e., is not as flat as for the longer chains) implying that $K_{\text {lin }}$ follows a length dependence law different from $1 / N$.

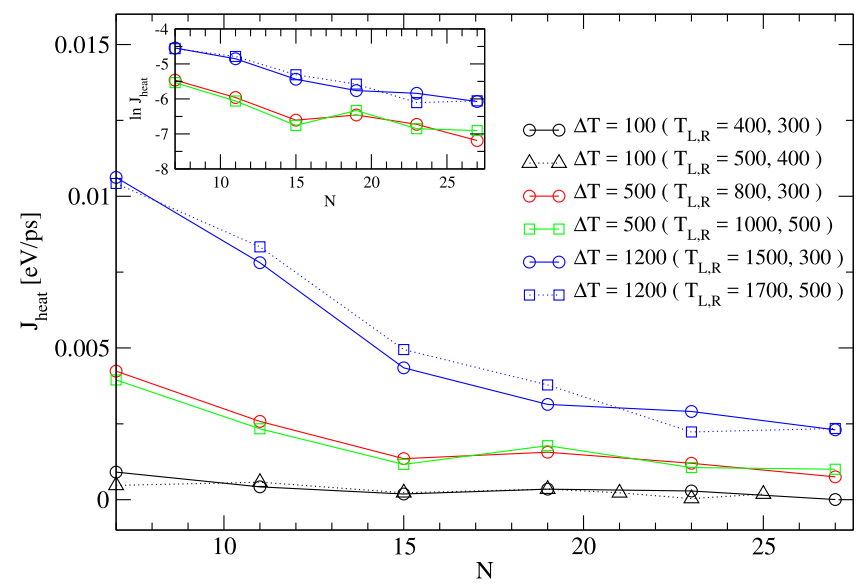

FIG. 4. Heat current $J_{\text {heat }}$ versus length $N$ of the chains for different sets of bath temperatures $T_{L}, T_{R}$. For small temperature differences $\Delta T \ll T_{L}, T_{R}$, the heat current is almost independent of the chain length (i.e., ballistic-like regime). For larger temperature differences $\Delta T>T_{L}$ or $T_{R}, J_{\text {heat }}$ decreases with the chain lengths. This behaviour is more typical for the diffusive transport regime. The inset shows that $J_{\text {heat }}$, on a logarithmic scale, decreases continuously with increasing $N$ and does not saturate for longer chains.

The dependence of the heat current $J_{\text {heat }}$ versus length $N$ for different sets of baths' temperatures $T_{L}, T_{R}$ is shown in Figure 4. For large temperature differences $\Delta T>T_{L}$ or $T_{R}$, the current $J_{\text {heat }}$ decreases with the chain length which is a characteristic of a diffusive system. For such large temperatures and temperature differences, one should keep in mind that the system is well beyond the linear response regime, and full non-linear and nonequilibrium effects are present. Determining the true nature of such non-linear effects requires further investigations which are beyond the scope of the present paper. For small temperature differences $\Delta T \ll T_{L}, T_{R}$, the heat current appears almost independent of the chain length (such a behaviour would characterise a ballistic transport regime). The inset in Figure 4 also clearly shows, on a logarithmic scale, that $J_{\text {heat }}$ does not saturate for the longer chains, especially for larger temperatures and temperature differences (the blue curves in Fig. 4).
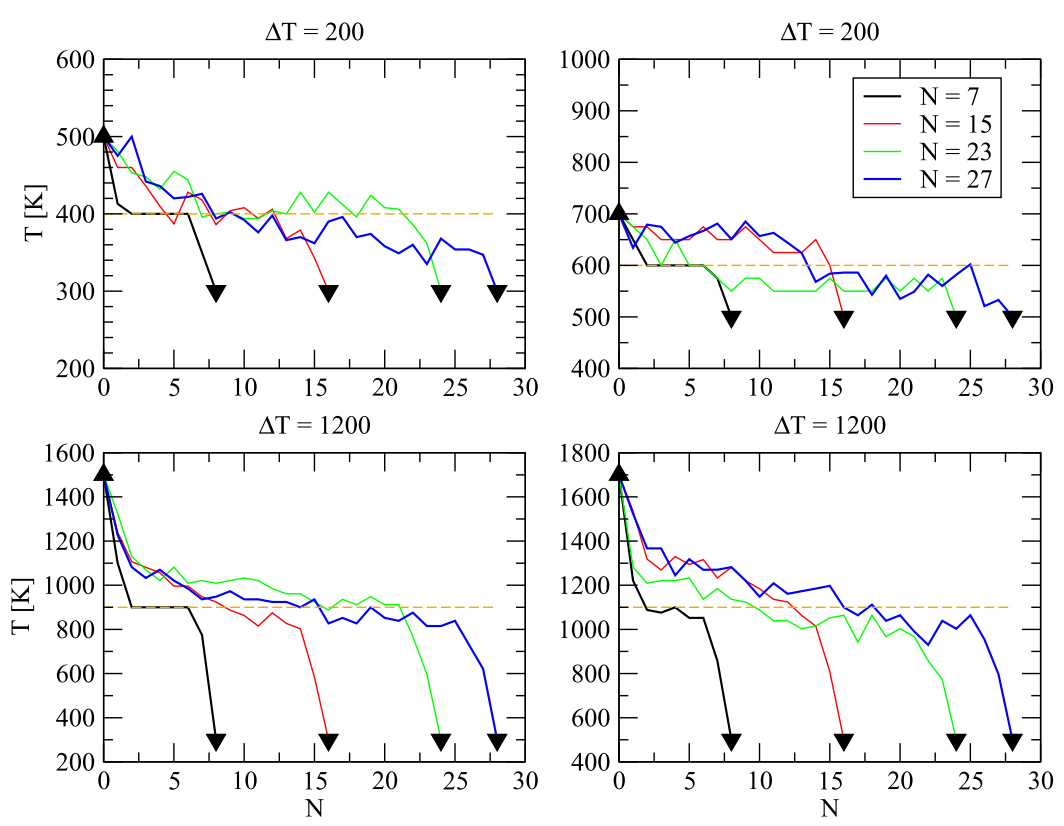

FIG. 5. Temperature profiles of 1D chains of different lengths $N=7,15,23$, and 27 for different bath temperatures $T_{L}$ (filled upward triangle) and $T_{R}$ (filled downward triangle). For the shortest chain $(N=7)$, the temperature profile is always flat (except for the large temperature difference when $T_{L}=1700$ and $T_{R}=500$ ) and is characteristic of the ballistic transport. For the longest chain $N$ $=27$, the temperature profile in the chain always presents a gradient even for $T_{L}=500$ and $T_{R}=300$, characteristic of the diffusive transport regime (Fourier law). An intermediate behaviour is obtained for the other chains $N$ $=15,23$. 
Before we can establish the nature of the transport regime, we now concentrate on the temperature profiles across the chains. As we mentioned in the Introduction, the diffusive transport regime is also associated with the establishment of a linear temperature profile across the system. The temperature profiles for different chain lengths and different temperature differences are shown in Figure 5. One can see the presence of a temperature gradient across the longer chains. The gradient becomes more pronounced for larger temperature differences. [Note that, for the systems considered here (one-dimensional chains connected to 3D baths), most of the temperature drop occurs at the contact between the chain and the bath (i.e., large thermal contact resistance) in contrast to systems with a larger (constant and finite) cross section.]

For shorter chains, apart from the case of a very large temperature difference $\left(T_{L}=1700, T_{R}=500\right)$, the temperature profile across the chain is always flat with the temperature given by $T_{\mathrm{av}}=\left(T_{L}+T_{R}\right) / 2$, see Fig. 5 . As we mentioned in the Introduction, this behaviour is a typical characteristic of the ballistic thermal transport regime. Therefore we can conclude that, by changing the temperature differences and/or the length of the system, we can obtain two different transport regimes.

The short chains appear to behave like a harmonic system with ballistic transport properties and no temperature gradient. For the longer chains and at high temperature differences, the dependence of $K_{\text {lin }}(N)$ and $J_{\text {heat }}(N)$ vs $N$ and the presence of a temperature gradient across the chain indicate a more diffusive transport regime. This could be a signature either of an anharmonicity in the systems or of the existence of localised vibration modes. Note that for small temperatures and small temperature differences, all chains appear to behave as ballistic harmonic systems.

It is important now to understand what kind of physics is behind the two transport regimes. For that we check first how the eigenmodes of vibration of the 1D chains change when $N$ increases. The eigenmodes of vibration of the chains are obtained from the diagonalisation of the dynamical matrix of the chains connected to the baths, as shown in Figure 1.

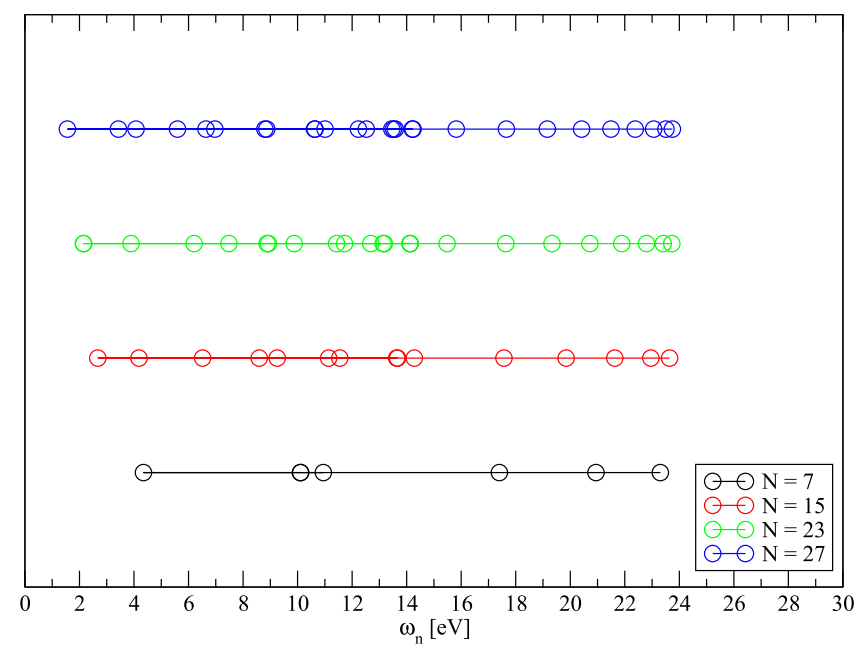

FIG. 6. Eigenvalues (in eV) of the vibrational modes in the chains with $N$ $=7,15,23,27$ (bottom to top lines with circle). More (nearly) degenerate modes exist in the longer chains. However the "accumulation" of a lot of long wave-length modes (around $\omega \rightarrow 0$ ) is not yet achieved for $N=27$. There are no obvious bound states outside the energy spectrum $(\omega \lesssim 1$ or $\omega \gtrsim 24 \mathrm{eV})$ that would correspond to more localised vibrational modes.

Figure 6 shows the eigenvalues for four different chain lengths. One observes more (nearly) degenerate modes in the longer chains and a larger number of long wavelength modes in the longer chains, as expected. Furthermore, we do not see any eigenvalues outside the energy spectrum (i.e., $\omega \lesssim 1$ or $\omega \gtrsim 24 \mathrm{eV}$ ) that could correspond to localised modes (i.e., bound states). The presence of such more localised modes (in the longer chains) would lead to the breakdown of the ballistic properties.

Another possible mechanism is related to anharmonic effects in the interatomic potential. In order to understand such effects, we first consider the work of Segal et al. ${ }^{13} \mathrm{In}$ that work, a simpler bath model and coupling to the bath were used in comparison with our GLE-2B. However, in their model calculations, the authors were able to modify the interatomic potential used for their description of the interaction in the 1D chains. It was found that for purely harmonic chains,
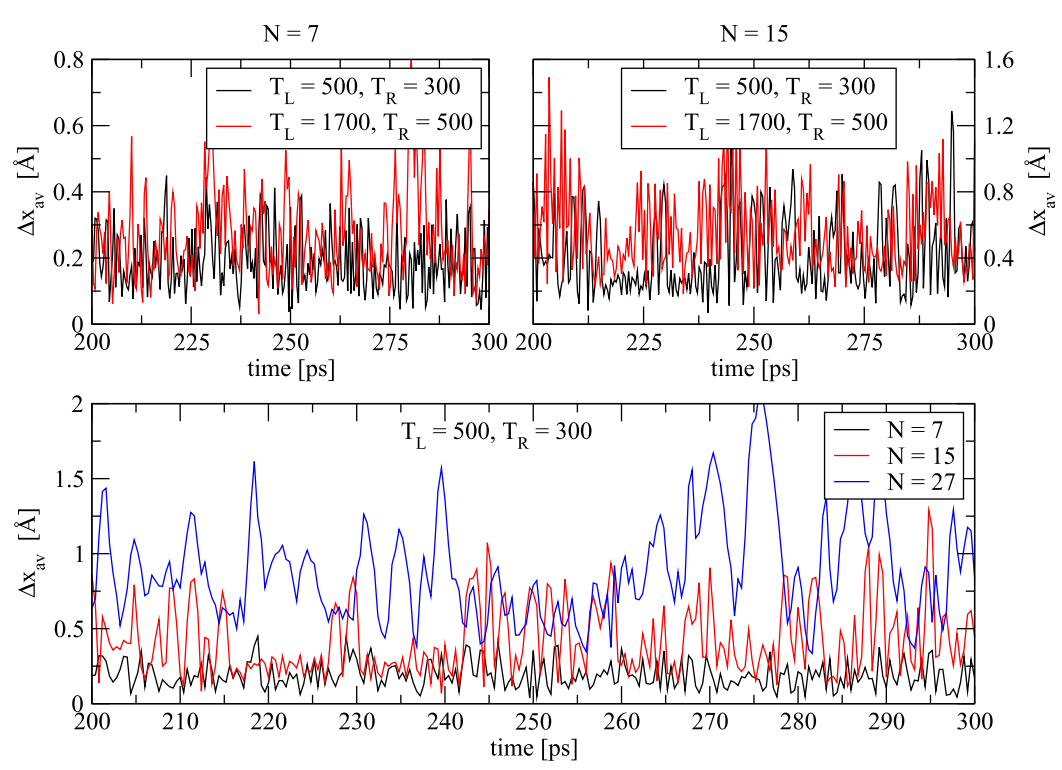

FIG. 7. Averaged displacement $\Delta x_{\mathrm{av}}$ versus time. The value of $\Delta x_{\mathrm{av}}$ increases when the temperature increases (see the two top panels). However such an increase is bigger when one increases the length of the chains. Hence in short chains, the atoms are more confined around the bottom of the potential energy wells, while for longer chains, the atoms have more "space" to move and are able to sample the anharmonic part of the potential well. 
the heat current is roughly independent of the chain length (a harmonic chain is an integrable model and presents ballistic properties no matter what the chain length is). When anharmonic effects are introduced in the interatomic potential, $J_{\text {heat }}$ becomes length dependent and decreases when the chain length increases (see Fig. 10 in Ref. 13). Such results are fully compatible with our calculations of $J_{\text {heat }}(N)$ shown in Figure 4.

In order to quantify the presence of the anharmonic effects, we consider the averaged atomic displacement $\Delta x_{\mathrm{av}}$. The averaged displacement $\Delta x_{\mathrm{av}}(t)$ is obtained from $\Delta x_{\mathrm{av}}(t)$ $=\left[\sum_{i=1}^{N}\left(\Delta x_{i}(t)\right)^{2} / N\right]^{1 / 2}$, where $\Delta x_{i}(t)$ is the relative displacement of atom $i$ in the chain of length $N$. The latter is calculated from $\Delta x_{i}(t)=x_{i}(t)-\left\langle x_{i}\right\rangle$, where $\left\langle x_{i}\right\rangle$ is the time averaged position of atom $i$ in the time window $\left[t_{\text {start }}, t_{\text {stop }}\right]$, i.e., $\left\langle x_{i}\right\rangle=\sum_{m=0}^{M} x_{i}\left(t_{\text {start }}+m \Delta t\right) /(M+1)$ with $t_{\text {stop }}=t_{\text {start }}+M \Delta t$.

Figure 7 shows the evolution of $\Delta x_{\mathrm{av}}$ versus time when the system has reached the steady state. The results show that, for a given chain length, the average variance $\Delta x_{\mathrm{av}}$ increases when the temperature increases, as expected (top panels in Fig. 7). However, for a given couple of temperatures $T_{L, R}$, the average variance is more pronounced in longer chains than in the shorter ones (see the bottom panel in Fig. 7). This suggests that for short chains, the atoms are more confined around the bottom of the potential energy wells, while for longer chains, the atoms have more "room" to move and are able to sample the anharmonic part of the potential energy well.

Fig. 8 shows typical configurations of the atoms in the long chain with $N=27$. Clearly, during the GLE run, some atoms get closer to each other (with distances smaller than the average interatomic distance), forming some kind of cluster. The distance between "clusters" is also larger than the average interatomic distance. Such a feature is also characteristic of the presence of long wavelength modes, which are more numerous in longer chains than in the shorter ones.

Therefore, we can conclude that, for longer chains and higher temperatures, the atomic motions sample a larger range of distances and a considerable part of the anharmonic EAM potential. This leads to the expected diffusive transport regime and to the presence of a temperature gradient across the long chains. However this behaviour is less apparent for low temperatures (as shown for example for $T_{L}=400$ and $T_{R}=300$ in Fig. 4).

For shorter chains, the motion of the atoms is more "restrained" and most probably their motion samples only the harmonic part of the potential. This leads to a more harmoniclike system with a more ballistic-like transport regime with

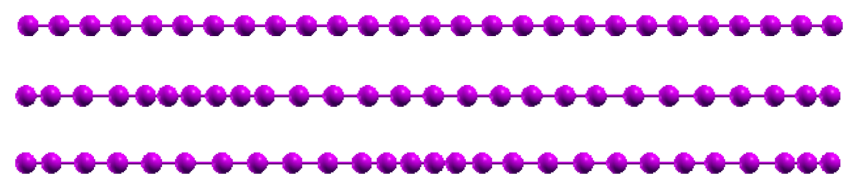

FIG. 8. Typical atomic configurations of the long chain with $N=27$ during a GLE run with $T_{L}=800$ and $T_{R}=300$. The configuration in the top panel corresponds to the initial conditions, with equally spaced atoms. During the GLE run, a form of clustering appears where the distances between atoms in (between) the clusters are smaller (larger) than the average interatomic distance, hence sampling the anharmonic parts of the potential. no temperature gradient across the short chains (except in the regime of very large temperature difference $\Delta T$ ). Note that such a behaviour for the atomic motion could also be "accentuated" by the fact that the three first (last) atoms of the chains are also directly interacting with the atomic baths.

\section{CONCLUSION}

We have used our recently developed generalised Langevin equation with 2 baths (GLE-2B) method $^{59}$ to study the thermal transport properties of $1 \mathrm{D}$ atomic chains coupled to two realistic 3D thermal baths kept at their own temperature. The results presented in this paper should be understood as a proof of principle of the robustness and efficiency of the numerical GLE-2B methodology that we have recently developed.

We have found that two different laws are obtained for the linear conductance versus the length of the chains. Furthermore, for large temperatures and temperature differences, the chains present a diffusive transport regime with the presence of a temperature gradient across the system. In such a regime, nonequilibrium effects are present and require an in-depth analysis. For lower temperatures and temperature differences, a regime reminiscent to the ballistic regime is observed. In short chains, except for the largest temperature differences considered, the temperature profile does not present any gradient, a characteristic of a ballistic transport property. Our detailed analysis suggests that the increase in anharmonic effects at higher temperatures/temperature differences is mainly responsible for the diffusive transport regime in the longer chains.

\section{ACKNOWLEDGMENTS}

We acknowledge financial support from the UK EPSRC, under Grant No. EP/J019259/1.

\section{APPENDIX A: CALCULATION OF THE HEAT CURRENT}

For the systems considered here, the Hamiltonian of the central region is given by

$$
H_{C}=\sum_{i \alpha} \frac{1}{2 m_{i}} p_{i \alpha}^{2}+V\left(\left\{r_{i \alpha}\right\}\right),
$$

where $p_{i \alpha}$ and $r_{i \alpha}$ are the momentum and position of the DOFs of the central region. The Hamiltonian $H_{v}$ of the harmonic bath $v=1,2$ is given by

$$
H_{v}=\sum_{b_{v}} \frac{1}{2 \mu_{l_{v}}} p_{b_{v}}^{2}+V_{(v)}^{\text {harm }}\left(\left\{u_{b_{v}}\right\}\right)
$$

with $p_{b_{v}}$ and $u_{b_{v}}$ being the momentum and position of the DOFs of the bath $v$, and $V_{(v)}^{\text {harm }}$ is the harmonic potential energy of the bath. The coupling between the bath $v=1,2$ and the central region is given by

$$
H_{v C}=\sum_{b_{v}} \mu_{l_{v}} f_{b_{v}}\left(\left\{r_{i \alpha}\right\}\right) u_{b_{v}},
$$


where the coupling force $f_{b_{v}}\left(\left\{r_{i \alpha}\right\}\right)$ between the central system and the bath $v$ is arbitrary with respect to the central system DOF.

In Refs. 54, 57, and 59, it was shown that the equation of motion (EOM) of the DOFs in the bath is given by

$$
\mu_{l_{v}} \ddot{u}_{b_{v}}=-\frac{\partial V_{(v)}^{\mathrm{harm}}}{\partial u_{b_{v}}}-\mu_{l_{v}} f_{b_{v}}\left(\left\{r_{i \alpha}\right\}\right) .
$$

The EOM for the DOF in the central region is given by

$$
m_{i} \ddot{r}_{i \alpha}=-\frac{\partial V(\mathbf{r})}{\partial r_{i \alpha}}-\sum_{\nu=1}^{2} \sum_{b_{\nu}} \mu_{l_{\nu}} g_{i \alpha, b_{\nu}} u_{b_{\nu}},
$$

where $g_{i \alpha, b_{\nu}}=\partial f_{b_{v}} / \partial r_{i \alpha}$.

Now, we define the heat current $J_{v}$ flowing between the central region and the bath $v=1$ as follows:

$$
J_{v}=\frac{d}{d t}\left(H_{v}+H_{v C}\right) \text {. }
$$

This definition arises from the local continuity equation (between the Hamiltonian density and the corresponding flux $^{3}$ ) integrated over the volume encompassing the bath $v$ DOFs. The volume integration of the time derivative of the Hamiltonian density gives the RHS of Eq. (A6). The volume integration of the divergence of the flux is transformed into a surface integral of the flux over the interface between the bath $v$ and the central region. The latter surface integral (with the surface normal vector pointing from the bath towards the central region) provides the total heat current $J_{v}$ flowing through the interface between the bath $v$ and the central region.

Using elementary calculus $(d H(p, q) / d t=\dot{p} d H / d p$ $+\dot{q} d H / d q)$ and from the definition of $H_{v}$ and $H_{v C}$, one finds that

$$
\begin{aligned}
J_{v}= & \sum_{b_{v}} \dot{u}_{b_{v}}\left(\mu_{l_{v}} \ddot{u}_{b_{v}}+\frac{\partial V_{(v)}^{\mathrm{harm}}}{\partial u_{b_{v}}}\right)+\mu_{l_{v}} f_{b_{v}} \dot{u}_{b_{v}} \\
& +\mu_{l_{v}} u_{b_{v}} \sum_{i \alpha} g_{i \alpha, b_{v}} \dot{r}_{i \alpha} .
\end{aligned}
$$

By using the EOM Eq. (A4) of the bath DOF, the above equation reduces to

$$
J_{v}=\sum_{i \alpha} \dot{r}_{i \alpha}\left(\sum_{b_{v}} \mu_{l_{\nu}} g_{i \alpha, b_{v}} u_{b_{v}}\right) .
$$

Note that, by definition, $\mu_{l_{v}} g_{i \alpha, b_{v}}$ is the spatial derivative of the force between the DOFs $i \alpha$ and $b_{v}$, and $u_{b_{v}}$ are small displacements of the bath DOFs around their equilibrium positions. Therefore the quantity $\mu_{l_{\nu}} g_{i \alpha, b_{v}} u_{b_{v}}$ is a force and its sum $\mathcal{F}_{i \alpha}^{(v)}=\sum_{b_{\nu}} \mu_{l_{\nu}} g_{i \alpha, b_{\nu}} u_{b_{\nu}}$ can be seen as the total force acting on the DOF $i \alpha$ due to its coupling to the bath $v$. Consequently the hear current can be expressed as the sum of the products of velocity times force,

$$
J_{\text {heat }}=\sum_{i \alpha} \dot{r}_{i \alpha} \mathcal{F}_{i \alpha}^{(v)}
$$

For our 1D system, Eq. (A8) simply reduces to $J_{\text {heat }}$ $=\sum_{i} \dot{x}_{i} \mathcal{F}_{i x}^{(v)}$.
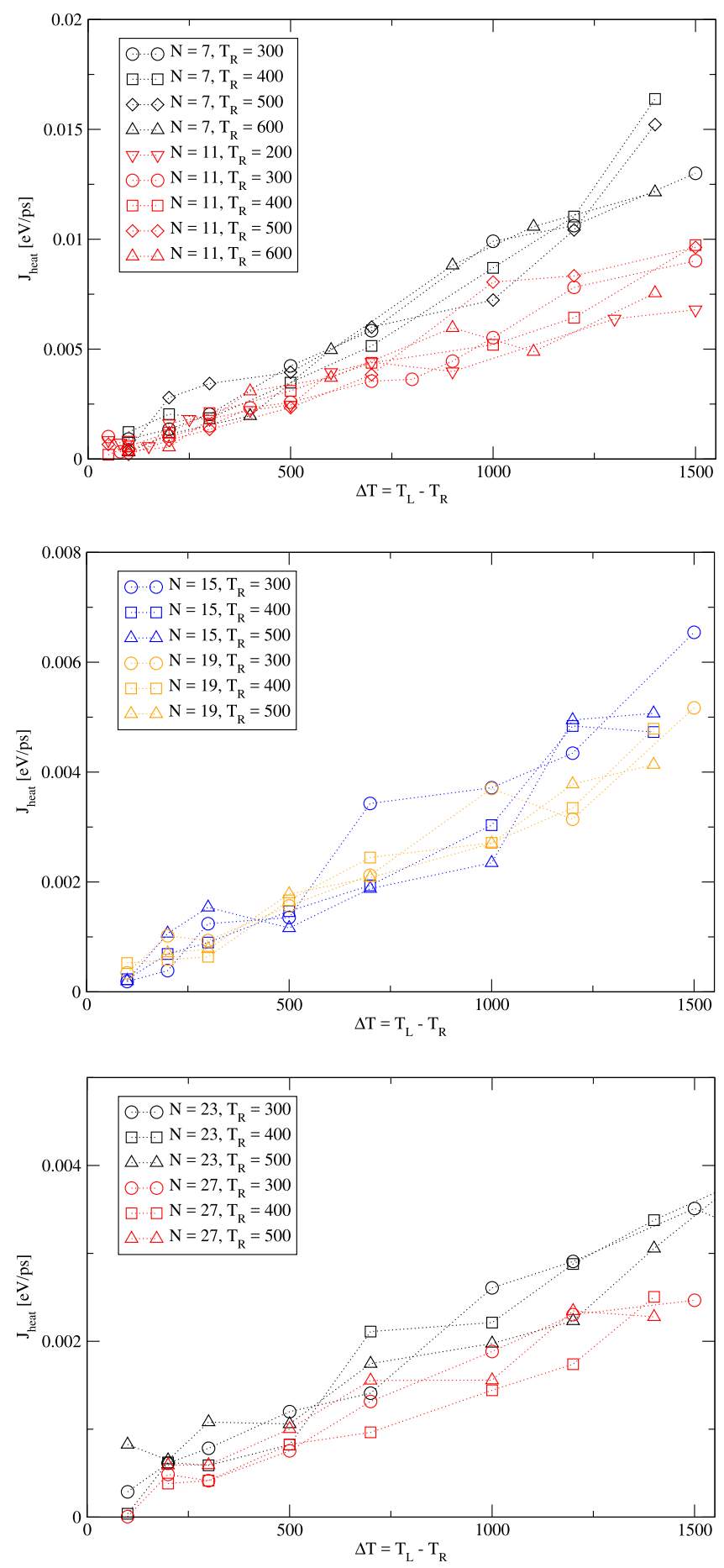

FIG. 9. Compilation of most of the results for the heat current $J_{\text {heat }}$ through the system, 1D chains of different length $N=7,11,15,23,27$ and for different baths' temperatures $T_{L}$ and $T_{R}$. Here the temperature difference is $\Delta T$ $=T_{L}-T_{R}$.

In the steady-state, the current is supposed to be the same (up to thermal fluctuations) in between any pair of atoms in the 1D chain. In practice, we calculate the heat current between each pair $(n, n+1)$ of atoms contained in the 1D chains. To make further connections with previous studies, ${ }^{3,13}$ we use the following notations: $\dot{x}_{n} \equiv v_{n}$ and $\mathcal{F}_{n x}^{(v)} \equiv f_{n}$. The heat current, between the pair $(n, n+1)$, is given by $j_{n, n+1}=\left(v_{n} f_{n}\right.$ $\left.+v_{n+1} f_{n+1}\right) / 2$, and we average $j_{n, n+1}$ over all pairs of atoms of the chain. 
We perform a further average over time, in the time range typically from 200 to $300 \mathrm{ps,}$, where the system has reached the steady-state to get the steady state heat current $J_{\text {heat }}$.

Note that when the force $f_{n}$ on atom $n$ in the chain is given by a (short-ranged) pairwise potential, it can be decomposed into two contributions from the nearest-neighbours, i.e., $f_{n}$ $=F_{n-1, n}+F_{n, n+1}$ with the nearest-neighbours forces $F_{n, m}$ $=F_{m, n}$. Our definition of the heat current $\sum_{n} v_{n} f_{n}$ becomes then equivalent (after a few manipulation of the indices $n-1$, $n, n+1)$ to the more commonly used expression for the current $\sum_{n}\left(v_{n+1}+v_{n}\right) F_{n+1, n}$ usually found in the literature. , $^{3,13}$

\section{APPENDIX B: HEAT CURRENT}

For information, we present in Figure 9 most of the results we have obtained for the heat current $J_{\text {heat }}$ flowing across all chains (length $N=7,11,15,19,23,27$ ) and for all sets of temperatures $T_{L}$ and $T_{R}$. From these results, it can be seen that the heat current increases with increasing temperature differences $\Delta T=T_{L}-T_{R}$ (as expected). Furthermore, $J_{\text {heat }}$ decreases when the length of the chain increases; such a behaviour is more pronounced for large temperature differences $\Delta T$.

Furthermore, we can estimate an error on the numerical values calculated for the heat current $J_{\text {heat }}$. We recall that the latter is calculated as an average of the heat current between the pairs $(n, n+1)$ in the chain,

$J_{\text {heat }}=\frac{1}{N_{\text {pair }}} \sum_{n=1}^{N-1}\left\langle j_{n, n+1}\right\rangle_{\tau}=\frac{1}{N_{\text {pair }}} \sum_{n=1}^{N-1}\left(\left\langle v_{n} f_{n}\right\rangle_{\tau}+\left\langle v_{n+1} f_{n+1}\right\rangle_{\tau}\right) / 2$.

The local heat current $\left\langle v_{n} f_{n}\right\rangle_{\tau}$ is obtained from the time average of $v_{n}(t) f_{n}(t)$ over the time period $t \in\left[t_{\text {start }}, t_{\text {end }}\right]$ with $t_{\text {end }}$ $-t_{\text {start }}=\tau$.

From the different simulations, we estimate an absolute error of $\Delta\left(j_{n, n+1}\right) \sim 0.008[\mathrm{eV} / \mathrm{ps}]$ for the local current. As the heat current $J_{\text {heat }}$ is an average over the different pairs, we assume that the corresponding standard error of the mean behaves as $\Delta\left(J_{\text {heat }}\right) \sim 0.008 / \sqrt{N_{\text {pair }}}[\mathrm{eV} / \mathrm{ps}]$.

Within this error margin, we can safely assume that the heat current calculated for $T_{L}=400$ and $T_{R}=300$ and for $T_{L}$ $=500$ and $T_{R}=400$ (shown in Fig. 4 by the black curves with circles and triangles, respectively) is nearly independent of the chain length.

${ }^{1}$ E. A. Jackson, J. R. Pasta, and J. F. Waters, J. Comput. Phys. 2, 207 (1968).

${ }^{2}$ H. Nakawa, Suppl. Prog. Theor. Phys. 45, 231 (1970).

${ }^{3}$ S. Lepri, R. Livi, and A. Politi, Phys. Rep. 377, 1 (2003).

${ }^{4}$ A. Dhar and D. Roy, J. Stat. Phys. 125, 801 (2006).

${ }^{5}$ A. Dhar, Adv. Phys. 57, 457 (2008).

${ }^{6}$ D. Segal and B. K. Agarwalla, Annu. Rev. Phys. Chem. 67, 185 (2016).

${ }^{7}$ Thermal Transport in Low Dimensions - From Statistical Physics to Nanoscale Heat Transfer, Notes in Physics Volume 921, edited by S. Lepri (Springer International Publishing, Switzerland, 2016).

${ }^{8}$ Z. Rieder, J. L. Lebowitz, and E. Lieb, J. Math. Phys. 8, 1073 (1967).

${ }^{9}$ M. Rich and W. M. Visscher, Phys. Rev. B 11, 2164 (1975).

${ }^{10}$ H. Spohn and J. L. Lebowitz, Commun. Math. Phys. 54, 97 (1977).

${ }^{11}$ G. Casati, Nuovo Cimento 52, 257 (1979).

${ }^{12}$ B. Hu, B. Li, and H. Zhao, Phys. Rev. E 61, 3828 (2000).

${ }^{13}$ D. Segal, A. Nitzan, and P. Hänggi, J. Chem. Phys. 119, 6840 (2003).

${ }^{14}$ D. Segal, J. Chem. Phys. 128, 224710 (2008).

${ }^{15}$ V. Kannan, A. Dhar, and J. L. Lebowitz, Phys. Rev. E 85, 041118 (2012).

${ }^{16}$ K. Sääskilahti, J. Oksanen, R. P. Linna, and J. Tulkki, Phys. Rev. E 86, 031107 (2012)
${ }^{17}$ G. T. Landi and M. J. de Oliveira, Phys. Rev. E 87, 052126 (2013).

${ }^{18}$ U. Zürcher and P. Talkner, Phys. Rev. A 42, 3278 (1990).

${ }^{19}$ K. Saito, S. Takesue, and S. Miyashita, Phys. Rev. E 54, 2404 (1996).

${ }^{20}$ K. Saito, S. Takesue, and S. Miyashita, Phys. Rev. E 61, 2397 (2000).

${ }^{21}$ A. Dahr and B. Sriram Shastry, Phys. Rev. B 67, 195405 (2003).

${ }^{22}$ C. Gaul and H. Büttner, Phys. Rev. E 76, 011111 (2007).

${ }^{23}$ A. Asadian, D. Manzano, M. Tiersch, and H. J. Briegel, Phys. Rev. E 87, 012109 (2013).

${ }^{24}$ R. J. Rubin and W. L. Greer, J. Math. Phys. 12, 1686 (1971).

${ }^{25}$ M. Bolsterli, M. Rich, and W. M. Visscher, Phys. Rev. A 1, 1086 (1970).

${ }^{26}$ H. Nakazawa, Prog. Theor. Phys. Suppl. 45, 231 (1970).

${ }^{27}$ A. M. Stoneham, Theory of Defects in Solids: Electronic Structure of Defects in Insulators and Semiconductors (Oxford University Press, Oxford, 1975), Chap. 11.

${ }^{28}$ J.-P. Eckmann, C.-A. Pillet, and L. Rey-Bellet, Commun. Math. Phys. 201, 657 (1999).

${ }^{29}$ T. Hatano, Phys. Rev. E 59, R1 (1999).

${ }^{30}$ Y. Zhang and H. Zhao, Phys. Rev. E 66, 026106 (2002).

${ }^{31}$ E. Pereira and R. Falcao, Phys. Rev. E 70, 046105 (2004).

${ }^{32}$ T. Mai and O. Narayan, Phys. Rev. E 73, 061202 (2006).

${ }^{33}$ J. Bricmont and A. Kupiainen, Commun. Math. Phys. 274, 555 (2007).

${ }^{34}$ T. Hu and Y. Tang, J. Phys. Soc. Jpn. 79, 064601 (2010).

${ }^{35}$ C. Giberti and L. Rondoni, Phys. Rev. E 83, 041115 (2011).

${ }^{36}$ E. Pereira, Physica A 390, 4131 (2011).

${ }^{37}$ T. N. Shah and P. N. Gajjar, Commun. Theor. Phys. 59, 361 (2013).

${ }^{38}$ G. P. Tsironis, A. R. Bishop, A. V. Savin, and A. V. Zolotaryuk, Phys. Rev. E 60, 6610 (1999).

${ }^{39}$ C. Kipnis, C. Marchioro, and E. Presutti, J. Stat. Phys. 27, 65 (1982).

${ }^{40}$ C. Bernardin and S. Olla, J. Stat. Phys. 121, 271 (2005).

${ }^{41}$ S. Lepri, C. Mejía-Monasterio, and A. Politi, J. Math. A: Math. Theor. 42, 025010 (2009).

${ }^{42}$ C. Bernardin, V. Kannan, J. L. Lebowitz, and J. Lukkarinen, J. Stat. Phys. 146, 800 (2012).

${ }^{43}$ J.-S. Wang and B. Li, Phys. Rev. E 70, 021204 (2004).

${ }^{44}$ P. Kim, L. Shi, A. Majumdar, and P. L. McEuen, Phys. Rev. Lett. 87, 215502 (2001).

${ }^{45}$ M.-H. Bae, Z. Li, Z. Aksamija, P. N. Martin, F. Xiong, Z.-Y. Ong, I. Knezevic, and E. Pop, Nat. Commun. 4, 1734 (2013).

${ }^{46}$ T.-K. Hsiao, H.-K. Chang, S.-C. Liou, M.-W. Chu, S.-C. Lee, and C.-W. Chang, Nat. Nanotechnol. 8, 534 (2013).

${ }^{47}$ S. Shen, A. Henry, J. Tong, R. Zheng, and G. Chen, Nat. Nanotechnol. 5, 251 (2010).

${ }^{48}$ D. Roy, Phys. Rev. E 77, 062102 (2008).

${ }^{49}$ T. Yamamoto, S. Konabe, J. Shiomi, and S. Maruyama, Appl. Phys. Express 2, 095003 (2009).

${ }^{50}$ D. Bagchi and P. K. Mohanty, J. Stat. Mech. 2014, P11025.

${ }^{51}$ M. S. Daw and M. I. Baskes, Phys. Rev. B 29, 6443 (1984).

${ }^{52}$ We have used the data file A199.eam.alloy from the NIST Interatomic Potential Repository Project (http://www.ctcms.nist.gov/potentials/) as provided by Ref. 53.

${ }^{53}$ Y. Mishin, D. Farkas, M. J. Mehl, and D. A. Papaconstantopoulos, Phys. Rev. B 59, 3393 (1999).

${ }^{54}$ L. Kantorovich, Phys. Rev. B 78, 094304 (2008).

${ }^{55}$ L. Kantorovich and N. Rompotis, Phys. Rev. B 78, 094305 (2008).

${ }^{56}$ S. J. Plimpton, J. Comput. Phys. 117, 1 (1995).

${ }^{57}$ L. Stella, C. D. Lorenz, and L. Kantorovich, Phys. Rev. B 89, 134303 (2014).

${ }^{58}$ H. Ness, L. Stella, C. D. Lorenz, and L. Kantorovich, Phys. Rev. B 91, 014301 (2015).

${ }^{59}$ H. Ness, A. Genina, L. Stella, C. D. Lorenz, and L. Kantorovich, Phys. Rev. B 93, 174303 (2016).

${ }^{60}$ R. Kupferman, J. Stat. Phys. 114, 291 (2004).

${ }^{61}$ J.-D. Bao, J. Stat. Phys. 114, 503 (2004).

${ }^{62}$ J. Łuczka, Chaos 15, 026107 (2005).

${ }^{63}$ M. Ceriotti, G. Bussi, and M. Parrinello, Phys. Rev. Lett. 102, 020601 (2009).

${ }^{64}$ M. Ferrario and P. Grigolini, J. Math. Phys. 20, 2567 (1979).

${ }^{65}$ F. Marchesoni and P. Grigolini, J. Chem. Phys. 78, 6287 (1983).

${ }^{66}$ C. C. Wan, J.-L. Mozos, J. Wang, and H. Guo, Phys. Rev. B 55, R13393(R) (1997).

${ }^{67}$ G. Taraschi, J.-L. Mozos, C. C. Wan, H. Guo, and J. Wang, Phys. Rev. B 58, 13138 (1998).

${ }^{68}$ J. Taylor, H. Guo, and J. Wang, Phys. Rev. B 63, 245407 (2001). 